\title{
Biomechanics of the rabbit knee and ankle: Muscle, ligament, and joint contact force predictions
}

\author{
Dustin M. Grover ${ }^{\mathrm{a}}$, Andrew A. Chen ${ }^{\mathrm{b}}$, Scott J. Hazelwood ${ }^{\mathrm{a}, \mathrm{c}, *}$ \\ ${ }^{a}$ Department of Orthopaedic Surgery, University of California, Davis, Orthopaedic Research Laboratories, \\ 4635 2nd Avenue, Room 2000, Sacramento, CA 95817, USA \\ ${ }^{\mathrm{b}}$ Department of Biological Systems Engineering, University of California, Davis, CA 95616, USA \\ ${ }^{\mathrm{c} B i o m e d i c a l ~ E n g i n e e r i n g ~ G r a d u a t e ~ G r o u p, ~ U n i v e r s i t y ~ o f ~ C a l i f o r n i a, ~ D a v i s, ~ C A ~ 95616, ~ U S A ~}$
}

\begin{abstract}
Mathematical models of small animals that predict in vivo forces acting on the lower extremities are critical for studies of musculoskeletal biomechanics and diseases. Rabbits are advantageous in this regard because they remodel their cortical bone similar to humans. Here, we enhance a recent mathematical model of the rabbit knee joint to include the loading behavior of individual muscles, ligaments, and joint contact at the knee and ankle during the stance phase of hopping. Geometric data from the hindlimbs of three adult New Zealand white rabbits, combined with previously reported intersegmental forces and moments, were used as inputs to the model. Muscle, ligament, and joint contact forces were computed using optimization techniques assuming that muscle endurance is maximized and ligament strain energy resists tibial shear force along an inclined plateau. Peak forces developed by the quadriceps and gastrocnemius muscle groups and by compressive knee contact were within the range of theoretical and in vivo predictions. Although a minimal force was carried by the anterior cruciate and medial collateral ligaments, force patterns in the posterior cruciate ligament were consistent with in vivo tibial displacement patterns during hopping in rabbits. Overall, our predictions compare favorably with theoretical estimates and in vivo measurements in rabbits, and enhance previous models by providing individual muscle, ligament, and joint contact information to predict in vivo forces acting on the lower extremities in rabbits.
\end{abstract}

Keywords: Rabbit model; Muscle; Knee ligament; Joint contact force; Optimization

\section{Introduction}

Knowledge of in vivo forces acting on the lower extremities is essential for developing theoretical and experimental animal models of musculoskeletal biomechanics and diseases (Burr et al., 1990; De Man et al., 2005; Lieberman et al., 2003; Richards et al., 1998; Yoshioka et al., 1996). One of the main challenges in theoretical biomechanical modeling is to resolve the redundancy problem in predicting muscle forces, which occurs with more muscles than equilibrium conditions at the joint (Crowninshield and Brand, 1981). Previous researchers using determinate models to predict knee muscle forces during walking in humans (Hurwitz et al., 1998; Morrison, 1968) and rabbits (Gushue et al., 2005) resolved redundancy by reducing individual muscles into functional groups and neglecting co-contraction.

Indeterminate models using static optimization criteria to resolve redundancy have been employed to estimate individual muscle forces during walking in humans (Crowninshield and Brand, 1981; Glitsch and Baumann, 1997), sheep (Taylor et al., 2006), dogs (Shahar and BanksSills, 2002, 2004), and cats (Prilutsky et al., 1997). Of the numerous criteria employed, the nonlinear criterion of maximum musculoskeletal endurance agrees more closely with electromyography (EMG) muscle activity patterns 


\section{Nomenclature}

$m \quad$ total number of muscle elements

$f_{i} \quad$ force magnitude of the $i$ th muscle element

$\mathrm{PCSA}_{i}$ physiologic cross-sectional area of the $i$ th muscle element

$f_{i} / \mathrm{PCSA}_{i}$ stress of the $i$ th muscle element

$\bar{M}^{\mathrm{k}} \quad$ intersegmental moment vector at the knee

$\bar{M}^{\mathrm{a}} \quad$ intersegmental moment vector at the ankle

$\bar{r}_{i}^{\mathrm{k}} \quad$ moment arm vector of the $i$ th muscle element originating from the knee $\hat{f}_{i}$

$\sigma$

$E_{j}$
$F^{\mathrm{JR}}$

$F_{x}^{\mathrm{JR}}$

$F_{y}^{\mathrm{JR}}$

$f_{j}$ moment arm vector of the $i$ th muscle element originating from the ankle

unit force vector of the $i$ th muscle element maximum physiologically achievable muscle stress, $35 \mathrm{~N} / \mathrm{cm}^{2}$ (Zajac, 1989) strain energy of the $j$ th ligament crossing the knee anterior-posterior shear component of the knee joint reaction force medial-lateral shear component of the knee joint reaction force force magnitude of the $j$ th ligament during walking than linear optimization criteria (Crowninshield and Brand, 1981). The goal of the present study was to expand the work of Gushue et al. (2005) by developing an indeterminate rabbit model that (1) separates functional muscle groups and solves for individual muscle forces crossing the knee and ankle, (2) solves for knee ligament forces with the assumption that ligaments resist tibial translation parallel to an inclined instead of perpendicular tibial plateau, and (3) solves for joint contact forces at the ankle as well as the knee.

\section{Methods}

\subsection{Model geometry}

Geometric data were obtained from the right hindlimbs of three skeletally mature New Zealand white rabbits $(4.1 \pm 0.5 \mathrm{~kg})$. Procedures were conducted with approval from the Animal Care and Use Committee at UC Davis. Each hindlimb was isolated and the skin removed. Muscles and ligaments crossing the knee and ankle were identified (Barone et al., 1973; Crum et al., 2003; Wingerd, 1985), marked at their origins and insertions, and removed. Additional points were marked to determine a tibial coordinate system (Fig. 1) (Grood and Suntay, 1983). Origin and insertion coordinates were determined with the hip, knee, and ankle flexed to $145^{\circ}, 45^{\circ}$, and $70^{\circ}$, respectively $\left(180^{\circ}=\right.$ full extension) (Beloozerova et al., 2003; Goslow et al., 1973; Mansour et al., 1998), to orient the hindlimb when external moments and forces were near their peak at approximately $20 \%$ of stance (Gushue et al., 2005). The limb position was assumed fixed for the entire stance phase of hopping as this potential source of variability has minimal effect on knee contact force predictions (Gushue et al., 2005).

\subsection{Force elements}

Muscles included in the model were those that crossed the knee and ankle (Figs. 1 and 2). Muscles with multiple origins or insertions were subdivided into separately acting elements (Brand et al., 1982), with the exception of the quadriceps muscles which were reduced to a single functional group (Figs. 1 and 2). Other force elements included the four knee ligaments, medial and lateral knee contact forces (KCFs), and an ankle contact force (ACF) (Figs. 1 and 2). Each KCF was assumed to act perpendicular to the medial and lateral tibial plateaus respectively along the $y$-axis at a distance $1 / 4$ of the tibial plateau width from the origin (Harrington, 1983). The ACF was assumed to act at the ankle joint center along the long axis of the tibia. Intersegmental forces and moments reported by Gushue et al. (2005) were used as model inputs, with the assumption that intersegmental axial moments and shear forces at the knee and ankle, and the abduction-adduction moment at the ankle, were negligible; and that the intersegmental axial ankle force approximated that of the knee.

\subsection{Computation of muscle, ligament, and joint contact forces}

Equilibrium equations were developed to solve the indeterminate model for the forces generated by muscles, ligaments, and joint contact at the knee and ankle from $0 \%$ to $100 \%$ of stance during hopping. All forces reported hereon are normalized by body weight $(4.1 \mathrm{~kg})$. To compute muscle forces, ligaments and articular surfaces were assumed to constrain axial-rotation at the knee and ankle, and abduction-adduction rotation at the ankle. Thus, muscles were required to generate two components of intersegmental moment at the knee (abduction-adduction and flexion-extension) and one at the ankle (flexion-extension). Muscle forces were computed as shown (Crowninshield and Brand, 1981):

objective function : $\min U=\sum_{i=1}^{m}\left(\frac{f_{i}}{\operatorname{PCSA}_{i}}\right)^{3}$,

constraints : $\quad 0 \leqslant \frac{f_{i}}{\operatorname{PCSA}_{i}} \leqslant \sigma$,

$\bar{M}^{\mathrm{k}}=\sum_{i=1}^{14} f_{i}\left(\bar{r}_{i}^{\mathrm{k}} \times \hat{f}_{i}\right)$

$\bar{M}^{\mathrm{a}}=\sum_{i=11}^{25} f_{i}\left(\bar{r}_{i}^{\mathrm{a}} \times \hat{f}_{i}\right)$

where PCSA values were taken from Lieber and Blevins (1989) with exception of the sartorius, which was assumed to be $66 \%$ of that of the gracilis (Sacks and Roy, 1982).

The knee joint reaction force (KJRF) was found as the magnitude of the vector subtraction of the resultant knee muscle force vector from the intersegmental knee force vector (Fig. 3). The $x$ and $z$ components of the KJRF were then transformed parallel and perpendicular to the tibial plateau, which sloped posterior and distal by $23 \pm 3^{\circ}$. The ankle joint reaction force (AJRF) was found as the magnitude of the vector subtraction of the resultant ankle muscle force vector from the intersegmental ankle force vector.

Knee ligament forces were predicted by resolving the shear component of the KJRF, assuming that translation of the femur stretches or slackens the ligaments. Thus, total ligament strain energy in tension approximates the work done by the shear force $\left(W^{\mathrm{JR}}\right)$. The required amount of translation $(\Delta x, \Delta y)$ was computed as:

objective function : $\min U=\sum_{j=1}^{4}\left(E_{j}\right)-W^{\mathrm{JR}}$,

constraints : $\quad F_{x}^{\mathrm{JR}} \Delta x \geqslant 0$ and $F_{y}^{\mathrm{JR}} \Delta y \geqslant 0$, 


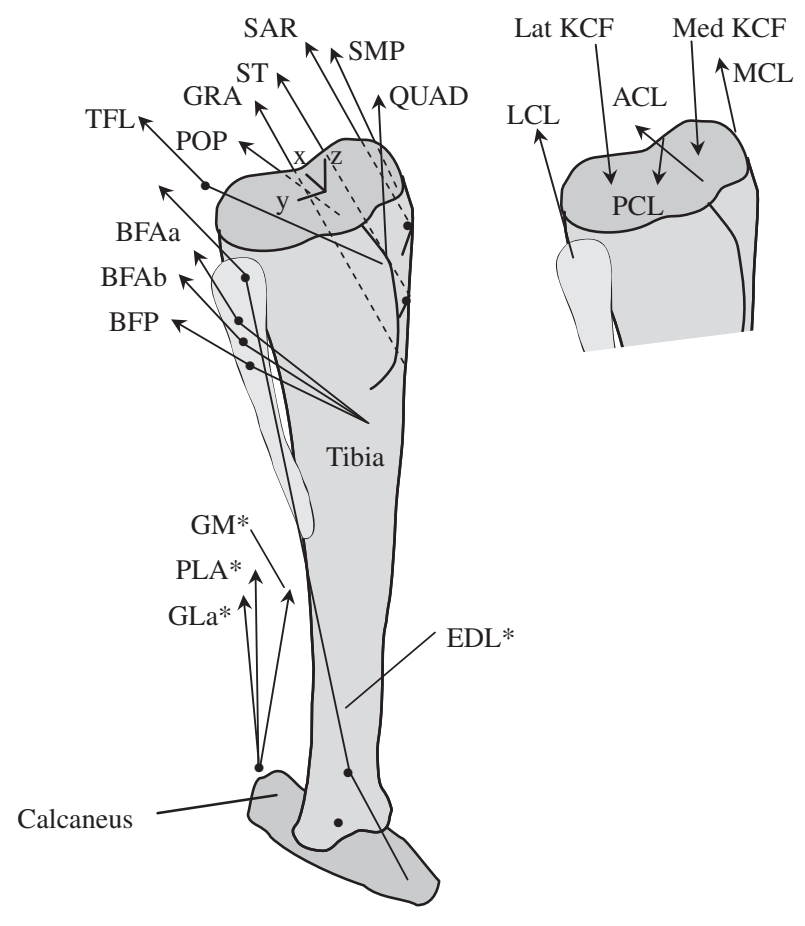

Fig. 1. A schematic diagram depicting the lines of action of muscle, ligament, and contact forces contributing to the intersegmental loads about the knee. $\mathrm{A} *$ indicates biarticular muscles crossing the knee and ankle. Muscle elements that combine to form the whole muscle with multiple origins or insertions are indicated with a lower case letter. For muscles not acting in a straight line between their origin and insertion, an attachment site was marked at the wrapping point (Brand et al., 1982) as indicated with a black dot. This was to avoid misrepresenting the moment arm, which could affect muscle force during hopping (Koh and Herzog, 1998). Hidden lines of action are indicated with a dashed line. The ankle joint center is indicated by a black diamond. The quadriceps (QUAD) muscles (i.e. rectus femoris, vastus medialis, vastus lateralis, and vastus intermedius) were reduced to a single functional group with its line of action along the patellar tendon (Gushue et al., 2005). The biceps femoris muscle is divided into three elements with a common insertion site on the tibia. These include a posterior head (BFP) and two anterior heads (BFAa, $\mathrm{BFAb}$ ). The lateral head of the gastrocnemius muscle is divided into two elements, one inserting on the lateral femoral condyle (GLa) and the other on the lateral tibial condyle (GLb) (see Fig. 2). The tensor fascia lattae (TFL) wraps over the LCL at the level of the joint line. The tibial coordinate system with origin placed at the center of the tibial plateau is also shown with the $z$-axis oriented proximally and passing midway between the two intercondyler eminences proximally and through the center of the ankle joint distally (midpoint between the malleoli). The $x$ axis is oriented posteriorly and defined as the cross product of the $z$-axis with a line connecting the center of each tibial plateau. The y-axis is oriented laterally and obtained by completing a right-handed coordinate system.

$f_{j} \geqslant 0$.

The KCF was found as the magnitude of the vector subtraction of the resultant knee ligament force vector from the KJRF vector (Fig. 3). The compressive KCF component was then divided into two elements, acting on the medial and lateral tibial plateaus, assuming that the medial KCF was $89 \%$ of that of the lateral (Gushue et al., 2005). The remaining KCF shear component was assumed to be distributed among the menisci and other soft tissues. The compressive and shear components of the ACF were assumed equal to those of the AJRF.

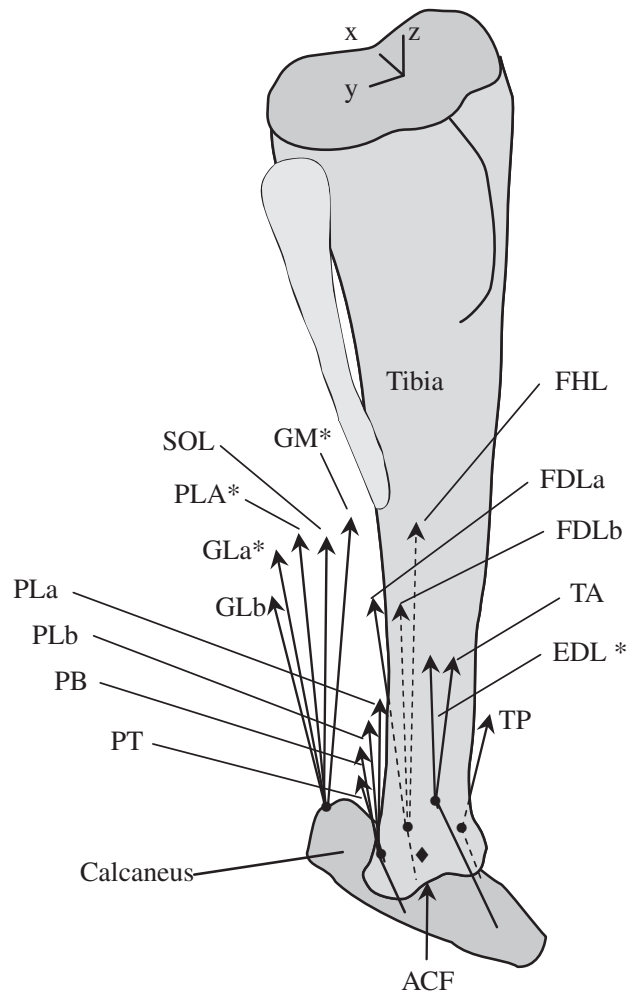

Fig. 2. A schematic diagram depicting the lines of action for muscle, ligament, and contact forces contributing to the intersegmental loads about the ankle. $\mathrm{A} *$ indicates biarticular muscles crossing the knee and ankle. Muscle elements that combine to form a muscle with multiple origins or insertions are indicated with a lower case letter. Wrapping points are indicated with a black dot. Hidden lines of action are indicated with a dashed line. The ankle joint center is indicated by a black diamond. The posterior aspect of the calcaneus bone serves as an insertion for the medial and lateral gastrocnemius and soleus muscles; and a wrapping point for the plantaris muscle.

\section{Results}

Our model predicted that both the gastrocnemius and plantaris, in addition to the quadriceps, contribute to the intersegmental knee moment during stance (Fig. 4), with synergistic load sharing seen between the gastrocnemius, plantaris, and soleus. Among the hamstrings, it was predicted that the biceps femoris produces the majority of load during stance (Fig. 5). In addition, the flexor digitorum longus, flexor hallucis longus, and peroneus muscles were shown to contribute along with the gastrocnemius, soleus, and plantaris to stabilize the ankle during stance (Fig. 6). The PCL and LCL were predicted to be the primary ligaments to stabilize the knee, with minimal load produced in the ACL and MCL during early stance (Fig. 7). The predicted resultant KCF was greater than the ACF, with more contact force exhibited during the early stages of stance.

\section{Discussion}

Overall, our muscle force predictions compare favorably with theoretical estimates and in vivo measurements in 
a

b

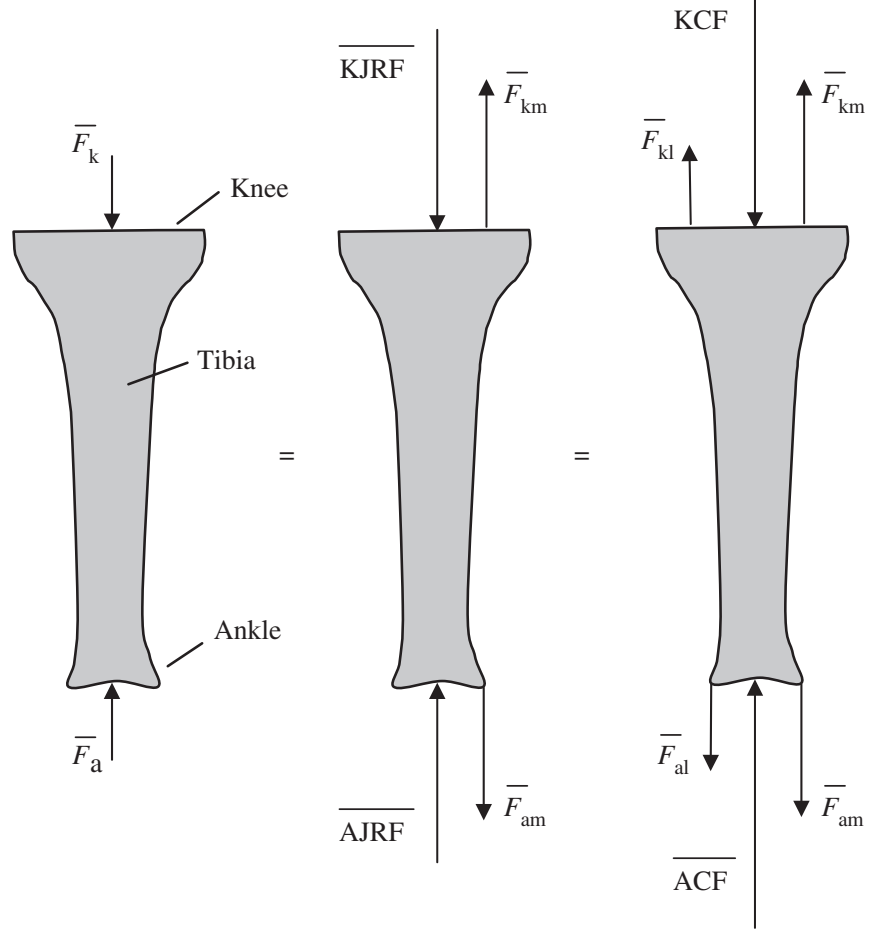

Fig. 3. Simplified free body diagrams showing the forces acting at the knee and ankle to create equilibrium of the tibia. Three equivalent loading conditions are shown to illustrate the distinction between the intersegmental force, joint contact force, and joint reaction force. Diagram (a) shows the intersegmental knee $\left(\bar{F}_{\mathrm{k}}\right)$ and ankle $\left(\bar{F}_{\mathrm{a}}\right)$ force vectors acting at the joint centers in compression during stance. Diagram (b) illustrates the joint reaction force and muscle force vectors and diagram (c) shows the joint contact force, muscle force, and ligament force vectors acting at the knee and ankle for equilibrium of the tibia during stance. The knee joint reaction force vector $(\overline{\mathrm{KJRF}})$ is found by vector subtraction of the knee muscle force vector $\left(\bar{F}_{\mathrm{km}}\right)$ from $\bar{F}_{\mathrm{k}}$. The knee contact force vector $(\overline{\mathrm{KCF}})$ is found by vector subtraction of the knee ligament force vector $\left(\bar{F}_{\mathrm{kl}}\right)$ from $\overline{\mathrm{KJRF}}$. Ankle force vectors are found in a similar manner.

rabbits. Our peak quadriceps force is between the theoretical estimate of Gushue et al. (2005) and the in vivo patellar tendon measurement of Juncosa et al. (2003), and our peak gastrocnemius force is slightly less than the theoretical estimate of Gushue et al. (2005) and the in vivo calcaneus tendon measurement by West et al. (2004). Differences between our force predictions and those of Gushue et al. (2005) might be due to our model using more muscles to balance the external knee moment.

Including deep knee flexion in our model produced a large shear component in the KJRF that resulted in a large PCL force and minimal ACL activity, in contrast to the estimate of Gushue et al. (2005). In addition, since normal ligament function is to guide the knee (Woo et al., 1990), minimal load was predicted for the MCL, which is consistent with the low loads measured in situ by Woo et al. (1990) and King et al. (1995). Also, the late rise in PCL force during weight bearing in our model is

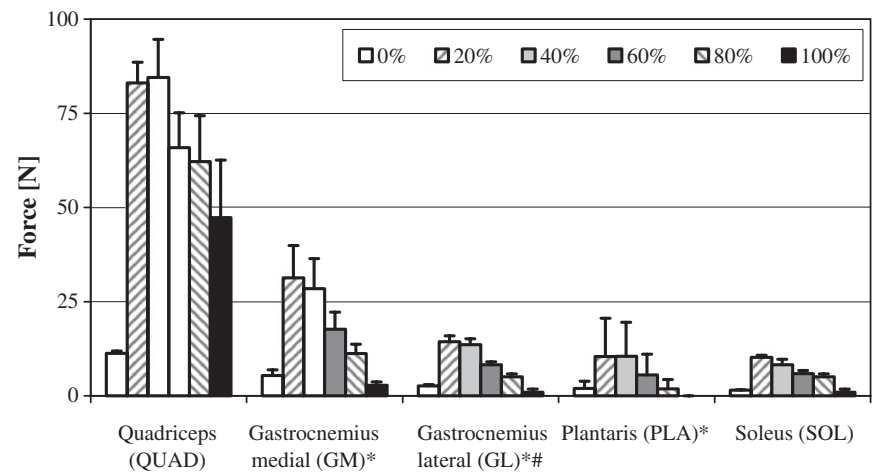

Fig. 4. Predicted muscle activity plotted at $20 \%$ intervals during the stance phase of hopping for the quadriceps (peak $84.5 \pm 10.1 \mathrm{~N}$ at $40 \%$ ), medial gastrocnemius (peak $32.0 \pm 8.8 \mathrm{~N}$ at $30 \%$ ), lateral gastrocnemius (peak $15.5 \pm 2.0 \mathrm{~N}$ at $30 \%$ ), plantaris (peak $11.3 \pm 10.9 \mathrm{~N}$ at $30 \%$ ), and soleus (peak $10.2 \pm 0.6 \mathrm{~N}$ at $20 \%$ ). Average values were determined by first solving for the forces for each of three specimens and then computing the average of the results. A * indicates biarticular muscles crossing the knee and ankle. A \# indicates muscles that were divided into two or more elements. Error bars represent +1 standard deviation about the mean.

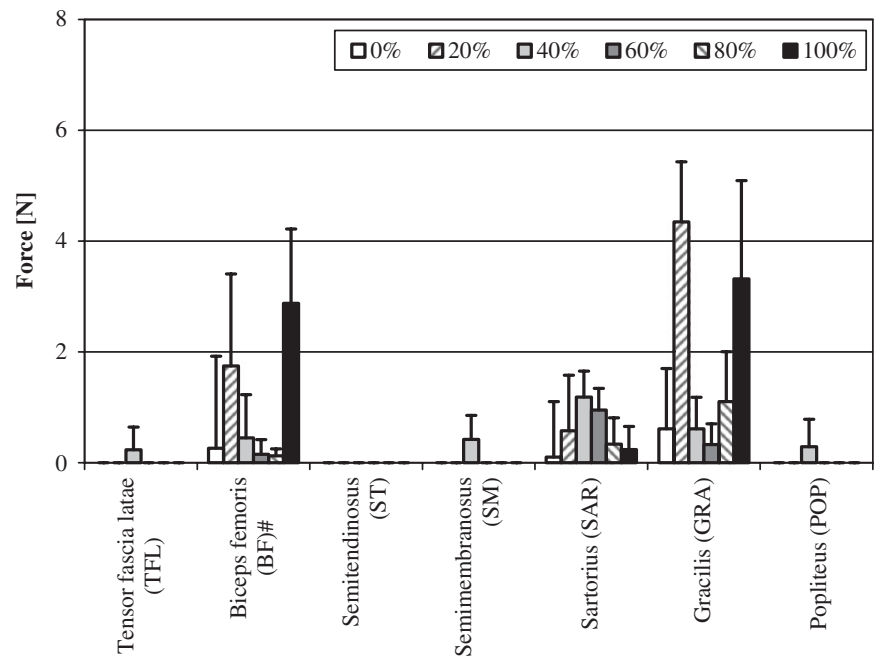

Fig. 5. Predicted muscle activity plotted at $20 \%$ intervals during the stance phase of hopping for tensor fascia latae (peak $0.2 \pm 0.4 \mathrm{~N}$ at $40 \%$ ), biceps femoris (peak $2.9 \pm 1.3 \mathrm{~N}$ at $100 \%$ ), semitendinosus, semimembranosus (peak $0.4 \pm 0.4 \mathrm{~N}$ at $40 \%$ ), sartorius (peak $1.2 \pm 0.5 \mathrm{~N}$ at $40 \%$ ), gracilis (peak $4.4 \pm 1.1 \mathrm{~N}$ at $20 \%$ ), and popliteus (peak $0.3 \pm 0.5 \mathrm{~N}$ at $30 \%$ ). No activity was predicted for semitendinosus. A \# indicates muscles that were divided into two or more elements. Error bars represent +1 standard deviation about the mean.

consistent with in vivo displacement of the tibia before the swing phase of hopping (Mansour et al., 1998). Our peak KCFs were lower than the theoretical estimates of Gushue et al. (2005), but greater than the in vivo measurements of Yang et al. (2004), which were made without muscle contraction, and Coughlin et al. (2005), whose results may have been influenced by altered joint kinematics caused by the transducer inserted in the tibial plateau. 


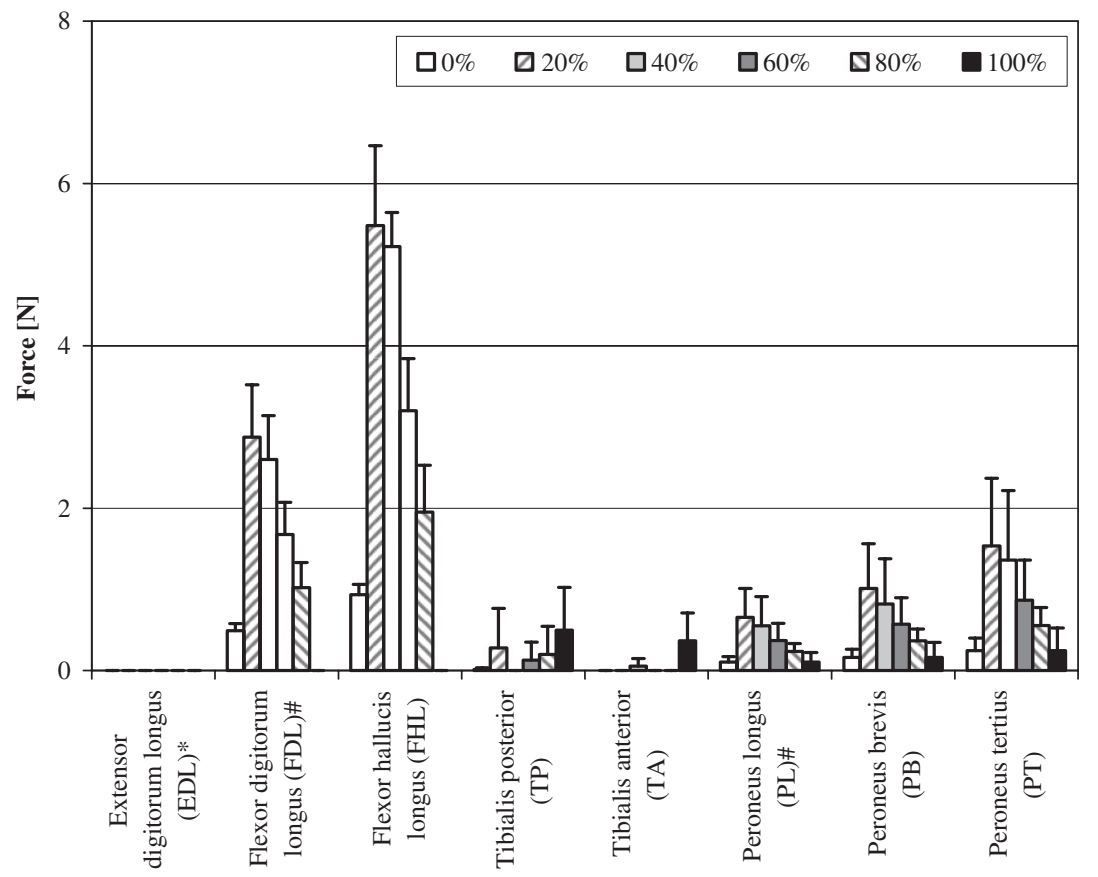

Fig. 6. Predicted muscle activity plotted at $20 \%$ intervals during the stance phase of hopping for extensor digitorum longus, flexor digitorum longus (peak $2.9 \pm 0.5 \mathrm{~N}$ at $30 \%$ ), flexor hallucis longus (peak $5.6 \pm 0.8 \mathrm{~N}$ at $30 \%$ ), tibialis posterior (peak $0.7 \pm 0.7 \mathrm{~N}$ at $90 \%$ ), tibialis anterior (peak $0.4 \pm 0.3 \mathrm{~N}$ at $100 \%$ ), peroneus longus (peak $0.7 \pm 0.4 \mathrm{~N}$ at $20 \%$ ), peroneus brevis (peak $1.0 \pm 0.5 \mathrm{~N}$ at $20 \%$ ), and peroneus tertius (peak $1.5 \pm 0.8 \mathrm{~N}$ at $20 \%$ ). No activity was predicted for extensor digitorum longus. A* indicates biarticular muscles crossing the knee and ankle. A \# indicates muscles that were divided into two or more elements. Error bars represent +1 standard deviation about the mean.

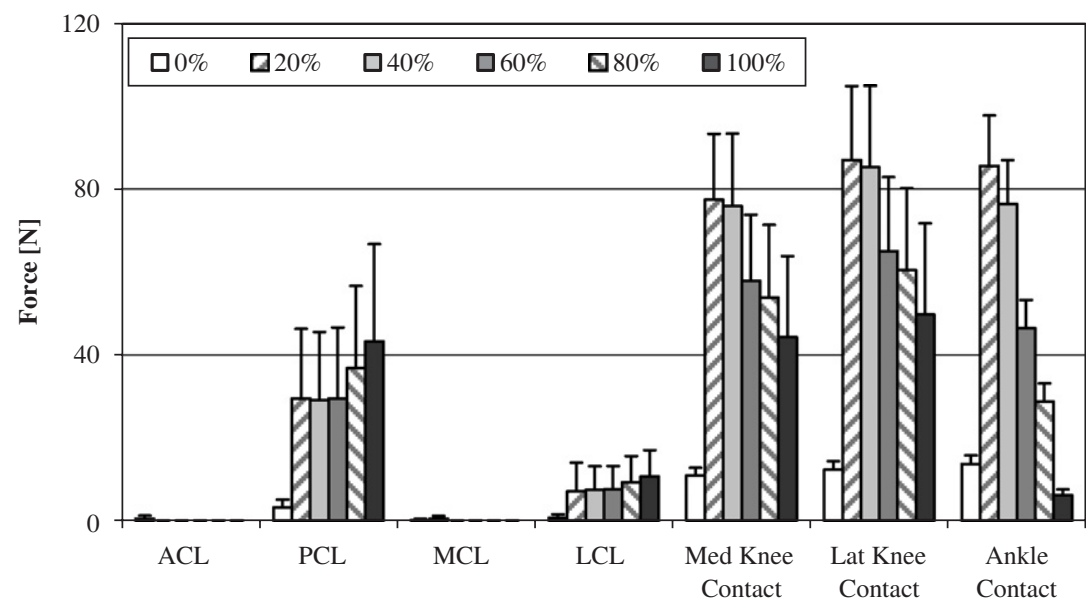

Fig. 7. Predicted knee ligament and joint contact forces (compressive components) acting at the knee and ankle plotted at $20 \%$ intervals during the stance phase of hopping. Peak forces in the anterior cruciate and medial collateral ligaments were $1.3 \pm 2.2$ and $1.1 \pm 0.6 \mathrm{~N}$ at $10 \%$ stance, respectively. Peak forces in the posterior cruciate and lateral collateral ligaments were $54.7 \pm 28.8$ and $13.6 \pm 8.4 \mathrm{~N}$ at $90 \%$, respectively. The peak medial and lateral KCFs were $77.5 \pm 15.8$ and $87.0 \pm 17.8 \mathrm{~N}$ at $20 \%$, respectively. The peak ACF was $86.4 \pm 12.3 \mathrm{~N}$ at $30 \%$ stance. Error bars represent +1 standard deviation from the mean.

One assumption of our study is that the inclusion of a more anatomically correct tibial plateau improves the model. To our knowledge, no previous small animal models have accounted for the inclination of the tibial plateau, which is well defined in rabbits (Crum et al., 2003). In a preliminary analysis, we discovered considerable differences in magnitude and direction of the anteriorposterior KJRF after adjusting the slope of the tibial plateau from $0^{\circ}$ to $24^{\circ}$, resulting in a decrease in the peak anterior-posterior KJRF of $72 \%$. This has a considerable effect on ligament forces.

One limitation of our study is that data used to validate the muscle and ligament forces were not related to joint kinematics during the stance phase of hopping, thus it is unknown when peak values occurred. Another limitation is that neither we nor Gushue et al. (2005) validated our 
models by comparing predicted force activity to EMG patterns in rabbits, although the low activity patterns in the TFL, SMP, POP, and EDL muscles in our rabbit model are very similar to patterns in the cat hindlimb during walking (Rasmussen et al., 1978). Nevertheless, the percentage of active muscles crossing the knee and/or ankle in our model ranges from $65 \%$ to $70 \%$ on average, which also is similar to a previous optimization model of the canine knee (Shahar and Banks-Sills, 2004).

In summary, our predictions provide a quantitative evaluation of force generated by individual muscles, ligaments, and joint contact during the stance phase of hopping. The data may be useful in theoretical rabbit models to study musculoskeletal biomechanics and diseases.

\section{Acknowledgments}

This work was supported by Whitaker Foundation Grant RG-02-0630.

\section{References}

Barone, R., Pavaux, C., Blin, P.C., Cuq, P., 1973. Atlas d' Anatomie du Lapin Atlas of Rabbit Anatomy. Masson, Paris, pp. 4-63.

Beloozerova, I.N., Zelenin, P.V., Popova, L.B., Orlovsky, G.N., Grillner, S., Deliagina, T.G., 2003. Postural control in the rabbit maintaining balance on the tilting platform. Journal of Neurophysiology 90, 3783-3793.

Brand, R.A., Crowninshield, R.D., Wittstock, C.E., Pedersen, D.R., Clark, C.R., Van Krieken, F.M., 1982. A model of lower extremity muscular anatomy. Journal of Biomechanical Engineering 104, 304-310.

Burr, D.B., Milgrom, C., Boyd, R.D., Higgins, W.L., Robin, G., Radin, E.L., 1990. Experimental stress fractures of the tibia. Biological and mechanical etiology in rabbits. Journal of Bone and Joint Surgery (Br) 72, 370-375.

Coughlin, K.M., Peura, G.D., Fleming, B.C., Hallock, S., Beynnon, B.D., 2005. In vivo loads in the medial compartment of the rabbit knee. Clinical Biomechanics 20, 1007-1009.

Crowninshield, R.D., Brand, R.A., 1981. A physiologically based criterion of muscle force prediction in locomotion. Journal of Biomechanics 14, 793-801.

Crum, J.A., Laprade, R.F., Wentorf, F.A., 2003. The anatomy of the posterolateral aspect of the rabbit knee. Journal of Orthopaedic Research 21, 723-729.

De Man, F.H., Tigchelaar, W., Marti, R.K., Van Noorden, C.J., Van Der Vis, H.M., 2005. Effects of mechanical compression of a fibrous tissue interface on bone with or without high-density polyethylene particles in a rabbit model of prosthetic loosening. Journal of Bone and Joint Surgery (Am) 87, 1522-1533.

Glitsch, U., Baumann, W., 1997. The three-dimensional determination of internal loads in the lower extremity. Journal of Biomechanics 30, $1123-1131$.

Goslow Jr., G.E., Reinking, R.M., Stuart, D.G., 1973. The cat step cycle: hind limb joint angles and muscle lengths during unrestrained locomotion. Journal of Morphology 141, 1-41.

Grood, E.S., Suntay, W.J., 1983. A joint coordinate system for the clinical description of three-dimensional motions: application to the knee. Journal of Biomechanical Engineering 105, 136-144.

Gushue, D.L., Houck, J., Lerner, A.L., 2005. Rabbit knee joint biomechanics: motion analysis and modeling of forces during hopping. Journal of Orthopaedic Research 23, 735-742.
Harrington, I.J., 1983. Static and dynamic loading patterns in knee joints with deformities. Journal of Bone and Joint Surgery (Am) 65, 247-259.

Hurwitz, D.E., Sumner, D.R., Andriacchi, T.P., Sugar, D.A., 1998. Dynamic knee loads during gait predict proximal tibial bone distribution. Journal of Biomechanics 31, 423-430.

Juncosa, N., West, J.R., Galloway, M.T., Boivin, G.P., Butler, D.L., 2003. In vivo forces used to develop design parameters for tissue engineered implants for rabbit patellar tendon repair. Journal of Biomechanics 36, 483-488.

King, G.J., Damson, E.L., Frank, C.B., Shrive, N.G., 1995. A new device and method for controlling the load in rabbit medial collateral ligament reconstructions. Journal of Biomechanical Engineering 117, 41-47.

Koh, T.J., Herzog, W., 1998. Excursion is important in regulating sarcomere number in the growing rabbit tibialis anterior. The Journal of Physiology 508 (Pt 1), 267-280.

Lieber, R.L., Blevins, F.T., 1989. Skeletal muscle architecture of the rabbit hindlimb: functional implications of muscle design. Journal of Morphology 199, 93-101.

Lieberman, D.E., Pearson, O.M., Polk, J.D., Demes, B., Crompton, A.W., 2003. Optimization of bone growth and remodeling in response to loading in tapered mammalian limbs. Journal of Experimental Biology 206, 3125-3138.

Mansour, J.M., Wentorf, F.A., Degoede, K.M., 1998. In vivo kinematics of the rabbit knee in unstable models of osteoarthrosis. Annals of Biomedical Engineering 26, 353-360.

Morrison, J.B., 1968. Bioengineering analysis of force actions transmitted by the knee joint. Bio-medical Engineering 3, 164-170.

Prilutsky, B.I., Herzog, W., Allinger, T.L., 1997. Forces of individual cat ankle extensor muscles during locomotion predicted using static optimization. Journal of Biomechanics 30, 1025-1033.

Rasmussen, S., Chan, A.K., Goslow Jr., G.E., 1978. The cat step cycle: electromyographic patterns for hindlimb muscles during posture and unrestrained locomotion. Journal of Morphology 155, 253-269.

Richards, M., Goulet, J.A., Weiss, J.A., Waanders, N.A., Schaffler, M.B., Goldstein, S.A., 1998. Bone regeneration and fracture healing. Experience with distraction osteogenesis model. Clinical Orthopaedics and Related Research 355 (Suppl), S191-S204.

Sacks, R.D., Roy, R.R., 1982. Architecture of the hind limb muscles of cats: functional significance. Journal of Morphology 173, 185-195.

Shahar, R., Banks-Sills, L., 2002. Biomechanical analysis of the canine hind limb: calculation of forces during three-legged stance. The Veterinary Journal 163, 240-250.

Shahar, R., Banks-Sills, L., 2004. A quasi-static three-dimensional, mathematical, three-body segment model of the canine knee. Journal of Biomechanics 37, 1849-1859.

Taylor, W.R., Ehrig, R.M., Heller, M.O., Schell, H., Seebeck, P., Duda, G.N., 2006. Tibio-femoral joint contact forces in sheep. Journal of Biomechanics 39, 791-798.

West, J.R., Juncosa, N., Galloway, M.T., Boivin, G.P., Butler, D.L., 2004. Characterization of in vivo achilles tendon forces in rabbits during treadmill locomotion at varying speeds and inclinations. Journal of Biomechanics 37, 1647-1653.

Wingerd, B.D., 1985. Rabbit Dissection Manual. The Johns Hopkins University Press \& The John Hopkins Press Ltd., Baltimore \& London, pp. 1-30.

Woo, S.L., Weiss, J.A., Gomez, M.A., Hawkins, D.A., 1990. Measurement of changes in ligament tension with knee motion and skeletal maturation. Journal of Biomechanical Engineering 112, 46-51.

Yang, L., Cai, G., Coulton, L., Saleh, M., 2004. Knee joint reaction force during tibial diaphyseal lengthening: a study on a rabbit model. Journal of Biomechanics 37, 1053-1059.

Yoshioka, M., Coutts, R.D., Amiel, D., Hacker, S.A., 1996. Characterization of a model of osteoarthritis in the rabbit knee. Osteoarthritis and Cartilage 4, 87-98.

Zajac, F.E., 1989. Muscle and tendon: properties, models, scaling, and application to biomechanics and motor control. Critical Reviews in Biomedical Engineering 17, 359-411. 